\title{
Multi-criteria decision algorithm for NQR signal detection
}

\author{
Cristian Monea \\ Doctoral School of Electronics, Telecommunications and \\ Information Technology \\ University Politehnica of Bucharest \\ Bucharest, Romania \\ cristianmonea0@gmail.com \\ Silviu Ionita \\ Faculty of Electronics, Communications and Computers \\ University of Pitesti \\ Pitesti, Romania \\ silviu.ionita@upit.ro
}

\author{
Gabriel V. Iana \\ Faculty of Electronics, Communications and Computers \\ University of Pitesti \\ Pitesti, Romania \\ gabriel.iana@upit.ro \\ Mihai Oproescu \\ Faculty of Electronics, Communications and Computers \\ University of Pitesti \\ Pitesti, Romania \\ mihai.oproescu@upit.ro
}

\begin{abstract}
Nuclear quadrupole resonance is a spectroscopy technique used for chemical analysis, temperature measurement and detection of prohibited substances. It offers advantages like highly specific and non-invasive detection, but has the disadvantages of low signal-to-noise ratio and temperature dependence of the response signal, which are generally compensated by special hardware and signal processing solutions. This paper proposes a data processing algorithm based on multiple criteria designed to ensure nuclear quadrupole resonance detection in specific scenarios. In contrast with current algorithms, this solution does not require statistical estimation of the response's parameters. It is evaluated using a real data set composed of 8000 acquisitions and is shown to reach a detection probability of $94 \%$ that outperforms existing solutions for a specific detection threshold.
\end{abstract}

Keywords-detection; multi-criteria; nuclear quadrupole resonance; signal-to-noise ratio; spectral diagram; statistical analysis

\section{INTRODUCTION}

Nuclear quadrupole resonance (NQR) is a solidstate radio frequency (RF) chemical analysis method used for substances that contain quadrupolar nuclei. Its working principle consists in exciting the substance with $\mathrm{RF}$ radiation and detecting the signal generated by its nuclei that absorb this radiation and release it in the form of a measurable RF signal when returning to the equilibrium state. The substance's response is highly dependent on its chemical structure and is used as a radio "fingerprint" for its detection [1], opening up this technique to a range of applications like temperature measurement, pharmaceutical and mineral analysis, and detection of dangerous and contraband substances [2].

From a signal processing standpoint, NQR detection presents the challenges of low signal-to-noise ratio (SNR) and susceptibility to RF noise and interferences (RFI), due to the very low substance response amplitude, which is in the order of tens of $n V$, comparable to the receiver's thermal noise level. Several signal processing solutions have been proposed to respond to these challenges and improve detection, ranging from energy detectors [3], that identify only one resonance frequency [4], to statistical algorithms [5-7]. The disadvantage of these solutions is their dependency on the estimation accuracy of the signal model's parameters which are used for taking the detection decision. A different approach has been proposed recently by using signal analysis techniques such as neural networks, support vector machines and decision trees to increase detection probability [8-10]. These approaches show good results, but their application to NQR detection is recent and further research is necessary to evaluate their performance in different detection scenarios.

This paper proposes a different approach from the solutions presented above by describing and evaluating a detection algorithm based on multiple criteria for improving the probability of detection. This solution does not require statistical estimation of the response's parameters. It uses several Fourier-based algorithms designed to offer advantages in specific detection scenarios and takes the detection decision by evaluating a weighted average of their results. The solution will be referred to as Multi-criteria Fourier-based Decision (MFD) algorithm. Multi-criteria algorithms have been proposed in numerous domains [11,12], but this solution is different because it is applied to NQR detection and uses criteria designed for this application.

The next section presents the theory of the proposed algorithm. In Section 3, we describe the experimental setup used to obtain the NQR data and in Section 4 we evaluate the proposed solution, discuss the results and perform a comparison with other algorithms. The last section summarizes the main results.

\section{THEORY OF THE Proposed AlgORITHM}

The MFD algorithm represents the fusion of six criteria (algorithms), which are based on statistical values (statistical detectors) and the maximum energy observed at a single frequency within the detection bandwidth (energy detectors). The detection decision is taken by evaluating a weighted average of the results. All signal processing is done in frequency domain, because the response can be observed more easily than in time domain, and all algorithms are based on the 
complex discrete Fourier transform (DFT) computed using the fast Fourier transform (FFT) algorithm.

The first algorithm initially assumes that the substance is detected and calculates the maximum peak amplitude in the spectrum. Then it checks if there is another spectral peak in the bandwidth around the central frequency, that exceeds an amplitude threshold. Considering the received signal vector $x[n]$ of length $N$, where $n$ is the sample index, then this algorithm concludes that no substance is detected if:

$$
\begin{gathered}
X[k] \geq A \\
k=\left\{i:-B \leq F(i) \leq B, i \neq k_{\text {max }}, X[i] \text { is peak }\right\}
\end{gathered}
$$

where $k$ is the set of searched indices,

$$
X[k]=\operatorname{DFT}\{x[n]\}
$$

$A$ is the peak amplitude threshold defined as a percentage $A_{p}$ of the maximum spectral peak's amplitude,

$$
A=A_{p} \max (\boldsymbol{X})
$$

$B$ represents the investigated bandwidth around the central frequency, also expressed as a percentage $B_{p}$ of the spectral width $\left(S_{W}\right)$ used at signal acquisition,

$$
B=B_{p} S_{W}
$$

$k_{\max }$ is the index of the spectral peak with the highest amplitude, and the function $F(i)$ returns the $k^{\text {th }}$ frequency bin. The condition $X[i]$ is peak is fulfilled if $X[i-1]<X[i]$ and $X[i+1]<X[i]$. A typical value used for $A_{p}$ and $B_{p}$ is 0.7 , and $S_{W}$ ranges from 50 to 200 $\mathrm{kHz}$.

As seen in Eq. (1), this algorithm checks all peaks inside the investigated bandwidth and if one (different that the maximum peak) is found having an amplitude at least equal to the threshold value, then it concludes that no valid response signal is present. This algorithm should allow identification of the NQR response in the presence of RFI that does not spectrally overlap the response.

The second algorithm is similar to the first one, with the difference that the bandwidth in which it searches for other peaks is centered on the maximum peak's frequency instead of the central frequency. This criterion should allow identification of the NQR response in low SNR and frequency offset scenarios (when the spectral peak may have an offset from the excitation frequency).

The third algorithm is different than the previous two, in the sense that it searches the entire spectrum. This criterion should be useful in identifying the NQR response in low SNR scenarios.

The fourth algorithm compares the spectrum's average value with an amplitude threshold that is defined as a percentage of the maximum spectral peak. It concludes that no substance is detected if:

$$
\bar{X}=\left(1 / N_{D F T}\right) \sum_{k=0}^{N_{D F T^{-1}}} X[k] \geq A
$$

where $N_{D F T}$ is the DFT's size. In this case, a typical value used for $A_{p}$ is 0.2 . This algorithm should allow identification of noise-only acquisitions when the average value is comparable with the maximum peak's amplitude. The purpose of the first four criteria is to determine if the acquired signal's spectrum contains the useful signal (NQR response) and noise, or only noise in case of noisy acquisitions, when the substance response is weak and surrounded by noise peaks.

The fifth algorithm processes the received signal by correlating it with a reference spectrum in order to identify the response spectral peak.

The spectrum is obtained by scanning a sodium nitrite sample in optimal (high SNR) conditions and has minimal noise and a high and clear response peak. This spectrum can be used as a reference for comparison, because all substances respond in a similar manner in good conditions. This algorithm calculates the Pearson correlation coefficient (PCC) and compares it with a correlation threshold, that is usually equal to 0.6 . This criterion should allow identification of NQR response in very low SNR scenarios.

The sixth algorithm compares the average values of the region around the frequency where the response peak is located and the side regions. In this regard, this algorithm would be useful in very noisy acquisitions, when the response peak is not visually identifiable in the spectrum, or in cases when the response linewidth is broadened due to non-uniform sample temperature. The central region is defined as a fixed bandwidth that is centered on the maximum peak's location. The average ratio threshold is typically equal to 3 .

The first three criteria are based on peak searching and comparing, and the last three criteria are based on statistical values. The final decision criterion (MFD), shown in Figure 1, is responsible for the fusion of all these algorithms and checks if the weighted average of the algorithms' results exceeds a predefined threshold, usually equal to 0.3 .

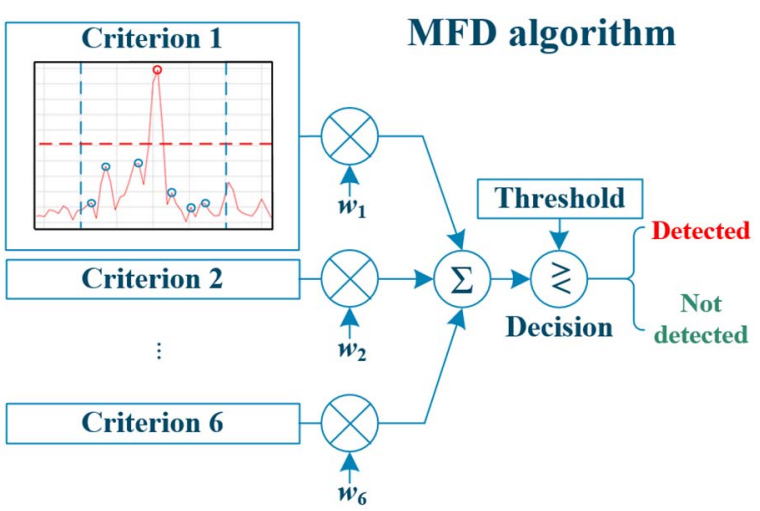

Figure 1. Illustration of the multi-criteria Fourier-based decision (MFD) algorithm

\section{EXPERIMENTAL SETUP AND PROCEDURE}

The experimental data is acquired using a custom designed NQR spectrometer [13]. The detection probe contains a planar coil on which the scanned item is placed and offers advantage of direct contact with the scanned object.

The NQR data is acquired from a $150 \mathrm{~g}$ sodium nitrite $\left(\mathrm{NaNO}_{2}\right)$ sample stored in a plastic bag. This substance presents a resonance frequency of 4.642 
$\mathrm{MHz}$ at around $24^{\circ} \mathrm{C}$, with a spin-lattice relaxation time of $100 \mathrm{~ms}$. The excitation frequency bandwidth is 2 $\mathrm{kHz}$, that should cover a temperature variation of approximately $\pm 1{ }^{\circ} \mathrm{C}$, taking into account that the frequency-temperature coefficient for this spectral line is $-1.6 \mathrm{kHz} /{ }^{\circ} \mathrm{C}$. The sweep is composed of 7 frequency steps, the acquisition window is $1300 \mathrm{~ms}$ and the spectral width is $185 \mathrm{kHz}$. Each data acquisition consists in 65 averaged samples per scan, acquired from 10 sequences composed of 100 scans (in total, 1000 averages per sample). The use of one substance to evaluate the MFD solution is considered sufficient since all substances respond in a similar manner, according to the NQR principle. The data samples are complex numbers resulting from a quadrature detection receiver. The data set used for evaluating the algorithm's performance consist in 8000 data acquisitions, where half of them are obtained in the presence of the substance (positive scans) and the rest are acquired in its absence (negative scans).

The evaluation procedure aims to acquire data in situations that match real detection scenarios. Therefore, the data acquisitions were realized in different conditions: (1) different distances between the sample and probe (placed directly over the probe, at 1 $\mathrm{cm}$ and at $5 \mathrm{~cm}$ ), (2) in the presence of sand, (3) with sample temperature variation and (4) with a slightly detuned resonant circuit. In the first case, the different distances from the sample and coil will enable the evaluation of the algorithm in different SNR conditions. In the second case, the algorithm is evaluated using acquisitions containing false response spectra due to piezoelectric noise caused by the inverse piezoelectric effect. In our case, we have used a sample containing sand and rocks. The data recorded in the third case will be used to show the performance in case of frequency offset; in this scenario, the substance's temperature is lowered a few degrees by temporarily storing it in a cooler room than that where the detector is located, before making the measurements. The last case is used for analyzing the algorithm in the case of false response spectral peaks that may appear when the resonant circuit is slightly detuned.

\section{RESULTS}

Figure 2 shows the acquired signals in the frequency domains, with the scanning scenario indicated in the upper right corner of the plots.

The MFD algorithm is based on six criteria, each with its own detection thresholds. After data processing, the weighted average of their decision is compared with a threshold in order to determine the final result. In order to allow selection of the thresholds and weights to ensure optimal detection, a receiver operating characteristic (ROC) analysis is performed on the data set by varying the threshold values from 0 to 100 ( $\%$ of highest peak's amplitude) for the first four criteria, 0 to 1 for the fifth criterion and 0 to 20 for the last criterion, resulting 15 data points.

The ROC analysis has shown that the last three criteria have better performance than the first three. This is caused by the fact that the peak search-andcompare method used by the first three criteria causes false alarms when noise or interference peaks have amplitudes close to that of the response.
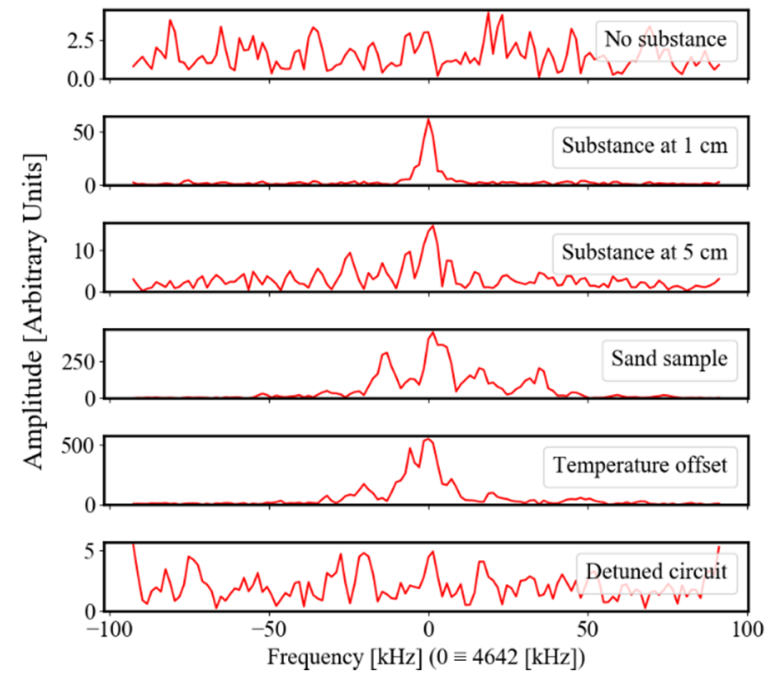

Figure 2. NQR response spectra in different detection conditions.

The area under the ROC curve indicator was calculated in each case and the optimal threshold value was determined, as shown in Table I.

TABLE I. ROC ANALYSIS RESULTS FOR THE CRITERIA USED BY THE MFD ALGORITHM

\begin{tabular}{|c|c|c|c|c|}
\hline $\begin{array}{c}\text { Criteria } \\
\text { no. }\end{array}$ & AUC $^{\mathbf{a}}$ & $\begin{array}{c}\text { Optimal } \\
\text { threshold }\end{array}$ & $\mathbf{P}_{\mathbf{d}}{ }^{\mathbf{b}}$ & $\mathbf{P}_{\mathbf{f}}^{\mathbf{c}}$ \\
\hline 1 & 0.71 & 78.5 & 0.68 & 0.26 \\
\hline 2 & 0.75 & 78.5 & 0.68 & 0.28 \\
\hline 3 & 0.77 & 78.5 & 0.68 & 0.24 \\
\hline 4 & 0.92 & 21.4 & 0.90 & 0.12 \\
\hline 5 & 0.90 & 0.57 & 0.93 & 0.13 \\
\hline 6 & 0.89 & 2.85 & 0.91 & 0.12 \\
\hline
\end{tabular}

Based on AUC indicator, the analysis indicates that criterion 4 provides the best detection performance and the statistical criteria have up to $21 \%$ better AUC values. However, the highest probability of detection is given by criteria 5 , for the threshold value of 0.57 . As expected, the first three criteria have the lowest classification performance. Taking into account the data presented in this table, the weights used by the MFD algorithm are assigned in the ascending order of the AUC values. After normalization, the weights vector is $[0.04,0.09,0.14,0.29,0.24,0.20]^{T}$.

Similarly, a 15-step ROC analysis was performed on the MFD algorithm in order to determine the decision threshold that ensures best detection performance. Since the generation of the ROC curve requires much time, the number of points was selected as the minimum value that can clearly show the evolution of the curve. The analysis indicates that the peak analysis degrades detection performance and the best detection is obtained using only statistical analysis. The optimal threshold is found to be 0.28 , providing a detection probability of $94 \%$. Furthermore, a confusion matrix analysis was performed in order to evaluate the statistical analysis MFD algorithm using the optimal values determined previously and its result is shown in Table II. 
TABLE II. CONFUSION MATRIX FOR THE MFD ALGORITHM BASED ON STATISTICAL ANALYSIS

\begin{tabular}{|c|c|c|}
\hline \multirow{2}{*}{ Predicted Class } & \multicolumn{2}{|c|}{ Actual Class } \\
\cline { 2 - 3 } & Substance present & Substance absent \\
\hline Detected & 3802 & 979 \\
\hline Not detected & 198 & 3021 \\
\hline
\end{tabular}

This analysis indicates the proportion of correct and incorrect classifications. Specifically, the number of true predictions represents around $85 \%$ of the total number of acquisitions, whereas the rest of $15 \%$ is the proportion of false detections. As required in real applications, the number of false negatives is the lowest.

A comparison was made with several recent classification algorithms [8-10], such as k-nearest neighbors (k-NN), support vector machine (SVM), neural network (NN) and ensemble of classifiers (EC). These solutions required data preparation and supervised learning in order to perform the analysis. The classifiers were implemented with the following characteristics: k-NN with 7 neighbors, SVM with polynomial kernel, NN with 11-5-1 neurons in inputhidden-output layers and 100 training epochs, and EC composed of 20 decision trees. The comparative analysis is performed on the same data set. The ROC curve for investigated algorithms is shown in Figure 3.

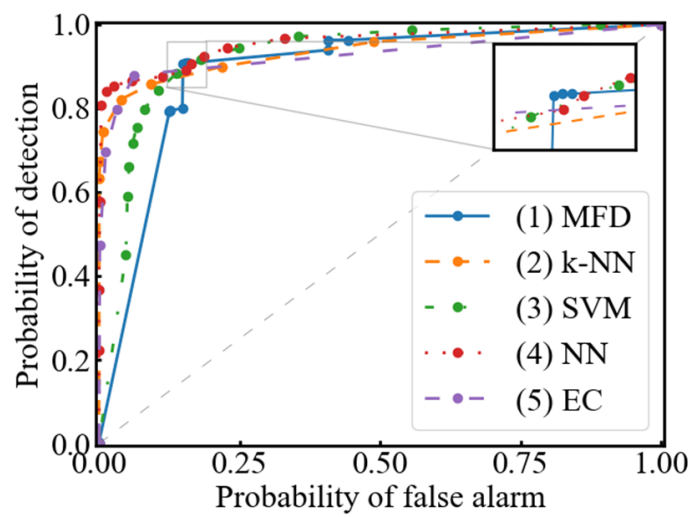

Figure 3. The ROC curves obtained for the MFD algorithm and several classifiers.

The MFD's curve has fewer discrete values than the other algorithms, which are concentrated in the regions with blue dots. As observed, MFD outperforms existing solutions by achieving a higher probability of detection for a specific threshold. Future research will focus applying machine learning techniques for signal analysis in order to increase true predictions and reduce false detections, as well as to discriminate the response signal from high noise components.

\section{CONCLUSIONS}

This paper describes a multi-criteria Fourier-based decision (MFD) algorithm for signal detection in NQR applications. The MFD algorithm is evaluated using experimental data acquired from a sodium nitrite sample in multiple detection scenarios. The use of one substance to evaluate the MFD solution is considered sufficient since all substances respond in a similar manner, according to the NQR principle. The optimal thresholds were determined for each criterion and used to establish the weights vector utilized by the MFD algorithm. ROC analysis has shown the optimal decision threshold to be 0.28 with a detection probability of $94 \%$, and that statistical analysis alone is sufficient to provide good detection performance. A comparison with existing solutions is performed and the proposed algorithm is shown to ensure a better probability of detection for a specific detection threshold. The application of machine learning techniques in NQR is proposed for improving detection using the statistical criteria as features for future algorithms.

\section{ACKNOWLEDGMENT}

This work is realized under Ph.D. stage at the Doctoral School of Electronics, Telecommunications and Information Technology, University Politehnica of Bucharest (Romania), contract number SD04/45/2018. This research is also supported by Mira Technologies Group (Romania).

\section{REFERENCES}

[1] C. Monea, "Techniques and equipment for detection of prohibited substances: A brief overview," J. Electrical Eng., Electronics, Control and Computer Science, vol. 4, no. 4, pp. 7-16, 2018.

[2] T. N. Rudakov, "Magnetic Resonance: NQR Technique and Instruments," Encyclopedia of Spectroscopy and Spectrometry, pp. 700-710, 2017.

[3] S. L. Tantum, et al., "Signal processing for NQR discrimination of buried land mines," Detection and Remediation Technologies for Mines and Minelike Targets IV, Aug. 1999.

[4] L. R. Cardona, Nuclear quadrupole resonance system for landmine detection in Antioquia, Ph.D. thesis, National University of Colombia, 2017.

[5] A. Jakobsson, M. Mossberg, M. D. Rowe, and J. A. S. Smith, "Exploiting temperature dependency in the detection of NQR signals," IEEE Transactions on Signal Processing, vol. 54, no. 5, pp. 1610-1616, May 2006.

[6] A. Jakobsson, M. Mossberg, M. D. Rowe, and J. A. S. Smith, "Frequency-selective detection of nuclear quadrupole resonance signals," IEEE Transactions on Geoscience and Remote Sensing, vol. 43, no. 11, pp. 2659-2665, Nov. 2005.

[7] S. D. Somasundaram, A. Jakobsson, and J. A. S. Smith, "Analysis of nuclear quadrupole resonance signals from mixtures," Signal Processing, vol. 88, no. 1, pp. 146-157, Jan. 2008.

[8] L. Cardona, H. Itozaki, J. Jiménez, N. Vanegas, and H. SatoAkaba, "Spectral descriptors and supervised classifier for ammonium nitrate detection in landmines by nuclear quadrupole resonance," Journal of Magnetic Resonance, vol. 305, pp. 104-111, Aug. 2019.

[9] Ibrahim, Parrish, Brown, and McDonald, "Decision Tree Pattern Recognition Model for Radio Frequency Interference Suppression in NQR Experiments," Sensors, vol. 19, no. 14, p. 3153, Jul. 2019.

[10] A. Nevzorov, A. Orlov, and D. Stankevich, "Machine learning in NQR TNT express detection system," Journal of Magnetic Resonance, vol. 308, p. 106596, Nov. 2019.

[11] R. Subbu, et al., "A Review of Two Industrial Deployments of Multi-criteria Decision-making Systems at General Electric," 2007 IEEE Symposium on Computational Intelligence in Multi-Criteria Decision-Making, Apr. 2007.

[12] A. Wiszniewski and B. Kasztenny, "A multi-criteria differential transformer relay based on fuzzy logic," IEEE Transactions on Power Delivery, vol. 10, no. 4, pp. 1786-1792, 1995.

[13] C. Monea, et al., "An optimized NQR spectrometer for detection of prohibited substances," Measurement, vol. 151, p. 107158, Feb. 2020. 